\title{
Standardization and decomposition of rates: Useful analytic techniques for behavior and health studies
}

\author{
JICHUAN WANG, AHMMED RAHMAN, HARVEY A. SIEGAL, and JAMES H. FISHER \\ Wright State University School of Medicine, Dayton, Ohio
}

\begin{abstract}
Standardization and decomposition are widely used analytic techniques in population studies for adjusting the impact of compositional factors on rates. This study demonstrates the application of these methods to behavior and health studies. Bootstrapping is used to estimate standard errors of the component effects and to conduct significance tests for them. The authors have developed a Windowsbased computer program that is demonstrated in the study for standardization and decomposition analysis by using empirical data on HIV seropositivity rates in two injection-drug-using populations in the northeastern United States.
\end{abstract}

When the rates of some phenomena between two populations or within the same population at two different time points are compared, the difference in the population composition should be taken into account. In population studies, standardization and decomposition of rates are commonly used techniques for adjusting the confounding effect of population composition on rates (Kitagawa, 1955, 1964; Pullum, 1978; United Nations, 1979). For example, it is possible for one population to have a crude death rate (the number of deaths occurring in a given year divided by the total population) that is lower than another's when the first population has higher age-specific death rates (i.e., the death rates for specific age groups). This paradox is a result of the fact that the first population has a considerably larger proportion of its population in age groups (e.g., ages 5-44) that are subject to lower death rates. This is similar to the example of Simpson's paradox provided by Bickel, Hammel, and O'Connell (1975) concerning the gender bias in acceptance rate to graduate school at Berkeley. Their study showed that females had a proportionately lower overall rate of acceptance because they applied disproportionately more often to the departments with the lowest rates of acceptance.

In the death rate example, the real difference between the crude death rates of the two populations is confounded by the difference in age structure between the two populations. Once age structure is standardized across the two populations, the adjusted death rate of the first popula-

This research was supported by the National Institute on Drug Abuse (Grant U01 DA07305). The authors thank the previous editor, Robert W. Proctor, reviewer Patrick Onghena, and another anonymous reviewer for their helpful comments on revision of the original manuscript. Correspondence concerning this article should be addressed to J. Wang, 143 Biological Sciences Bldg., Health Services Research Project, Substance Abuse Intervention Programs, School of Medicine, Wright State University, Dayton, OH 45435 (e-mail: jwang@wright.edu). tion would certainly be higher than that of the second population.

Standardization and decomposition are useful techniques for studying differences or changes in measures of behaviors and behavioral outcomes. To return to the death rate comparison example, on the basis of the results of standardization, the difference in crude death rates between the two populations can be decomposed into two component effects: (1) the component effect attributed to the difference in age structure, and (2) the rate effect attributed to the difference in the age-specific death rates. If another compositional factor (e.g., ethnicity) were taken into account, the difference in the crude death rate would be decomposed into three component effects: (1) the Factor 1 effect, which is due to the difference in age structure; (2) the Factor 2 effect, which is due to the difference in ethnic composition; and (3) the rate effect, which is due to the difference in the factor-specific (i.e., age- and ethnicityspecific) death rates.

Standardization and decomposition can be productively applied to behavior and health studies. In everyday life, many social, economic, political, and health behaviors, as well as the consequences or outcomes of these behaviors, are often measured by rate, percentage, or proportion. For example, the percentage of dirty-needle-sharing behavior among an injection drug user (IDU) population in a given time period is a measure of the prevalence rate of sharing contaminated needles in the population. To compare different prevalence rates between two IDU populations, the application of standardization and decomposition techniques will tell us how much of the observed difference can be attributed to the effects of composition factors, such as age, gender, and ethnicity, as well as the "real" difference in prevalence of risky needle behavior between the two IDU populations. Studies on differences in other behavior measures - such as the incidence rate of suicide or murder, the prevalence of school dropouts or substance abuse among students between 
gender or ethnic groups, the percentage or proportion of cigarette smoking or recreational physical exercise, and so on-between different populations can all be conducted in the same way.

Demographers have developed a variety of techniques for standardization and decomposition of rates. In general, the methods of standardization and decomposition are grouped into two broad categories (Das Gupta, 1991). In the first category, a crude rate is expressed as a function of one or several factors (Bongaarts, 1978; Nathanson \& Kim, 1989; Pullum, Tedrow, \& Herting, 1989; Wojtkiewicz, McLanahan, \& Garfinkel, 1990). In the second and more common category, standardization and decomposition are performed on cross-classified or contingency table data (Cho \& Retherford, 1973; Das Gupta, 1991, 1993; Kim \& Strobino, 1984; Kitagawa, 1955, 1964; Liao, 1989). In both categories, standardization and decomposition are usually performed on the basis of algebraic equations, rather than of statistical modeling. In a series of papers, Clogg and his colleagues (Clogg, 1978; Clogg \& Eliason, 1988; Clogg \& Shockey, 1985; Clogg, Shockey, \& Eliason, 1990) have developed a statistical model - the Clogg model - to standardize rates. Based on log-linear models, the Clogg model centers on the idea of purging the confounding effects of composition factors. However, the Clogg model is not designed for, and cannot be applied directly to, decomposition analysis. Liao (1989) has developed a method that applies the results of the Clogg models in such a way as to decompose the difference in crude rates into component effects representing composition effects, rate effect, and possible interactions between the two.

The choice of a decomposition method depends first on the type of data available for analysis; second, the choice of a method is a matter of personal preference. The often used standardization and decomposition methods in population studies are the ones that are based on algebraic presentation and cross-classified data format.

Unfortunately, for the analysis of sample data, none of the current decomposition methods take sampling variability into account. Although Liao's (1989) method is based on the results of statistical modeling, the actual calculation of the component effects is still based on algebraic equations. Therefore, like the other methods, it does not provide statistics for testing the significance of the component effects. In addition, with different purging methods, Liao's method provides slightly different component effects.

In this study, we introduce Das Gupta's (1991, 1993) standardization and decomposition method, which is performed on cross-classified data, to demonstrate the utilization of standardization and decomposition in the field of behavioral health studies. Like other conventional methods, Das Gupta's method has no constraints on the specification of relationship (e.g., linearity), the nature of the variables (e.g., random), or the form of variable distributions (e.g., normality). Unlike other conventional methods, Das Gupta's method integrates interactions between component effects into the additive main effects. This procedure makes interpretation of the results much easier, particularly when multiple factors are involved in the analysis.

After a brief description of Das Gupta's (1991, 1993) method, we will illustrate how standardization and decomposition can aid in comparative analysis of the HIV seropositivity rate-a measure of outcome that can result from health risk behaviors such as sharing dirty needles and unprotected sexual intercourse--between female and male IDU populations in the northeastern United States. In addition, we will briefly introduce a nonparametric method, known as bootstrapping, that can be applied to estimate the standard errors of the component effects. Therefore, we are able to conduct significance tests for the component effects.

\section{METHOD}

A total of 7,378 IDUs (1,745 females and 5,633 males) who took the voluntary and confidential HIV antibody tests in the National Institute on Drug Abuse's National AIDS Demonstration Research (NADR) projects between 1988 and 1991 (Brown \& Beschner, 1993) constitutes the sample for the study. We treat the female and male samples as two subpopulations: Female IDUs constitute Population 1, and male IDUs Population 2. For simplicity, only two compositional factors-Factor 1 (age structure) and Factor 2 (ethnic composition) - are taken into account for the analysis. The difference in the HIV seropositive rates between the male and the female IDU populations was decomposed into additive component effects: (1) the Factor 1 effect, attributed to different age structures; (2) the Factor 2 effect, attributed to different ethnic compositions; and (3) the rate effect, attributed to different factor-specific HIV seropositive rates.

\section{Notation and Algebraic Expression}

Population 1 (Female IDUs):

$N_{1 .} \quad$ Total number of female IDUs;

$N_{1 i}$. Number of female IDUs in the $i$ th category of Factor 1 (e.g., age, with four categories: 1: $<20$, 2: 20-29, 3: 30-39, and 4:40+);

$N_{1 . j} \quad$ Number of female IDUs in the $j$ th category of Factor 2 (e.g., ethnicity, with three categories: 1: Black, 2: Hispanic, and 3: White);

$N_{\mathrm{l} i j} \quad$ Number of female IDUs in the $i$ th category of Factor 1 and $j$ th category of Factor 2;

$T_{1 i j} \quad$ Age- and ethnicity-specific HIV seropositivity rate among the female IDUs in the $i$ th category of Factor 1 and the $j$ th category of Factor 2.

Population 2 (Male IDUs):

$N_{2}$. Total number of male IDUs; 
$N_{2 i} \quad$ Number of male IDUs in the $i$ th category of Factor 1 (e.g., age, with four categories: 1: $<20$, 2: 20-29, 3: 30-39, and 4:40+);

$N_{2 . j} \quad$ Number of male IDUs in the $j$ th category of Factor 2 (e.g., ethnicity, with three categories: 1: Black, 2: Hispanic, and 3: White);

$N_{2 i j} \quad$ Number of male IDUs in the $i$ th category of Factor 1 and the $j$ th category of Factor 2;

$T_{2 i j} \quad$ Age- and ethnicity-specific HIV seropositivity rate among the male IDUs in the $i$ th category of Factor 1 and the $j$ th category of Factor 2.

The crude HIV seropositivity rate for each of the two IDU populations is equal to the total number of HIV seropositives divided by the total number of IDUs in that population. The crude rates can be expressed as

$$
T_{1 . .}=\sum_{i j} \frac{T_{i j} N_{1 i j}}{N_{1 . .}}
$$

and

$$
T_{2 . .}=\sum_{i j} \frac{T_{2 i j} N_{2 i j}}{N_{2 . .}} .
$$

That is, the observed HIV seropositivity rate is a weighted summation of age- and ethnicity-specific HIV seropositivity rates in each population. The weights are the relative age-ethnicity frequencies or cell proportions in the contingency table - the ratios of $N_{1 i j} / N_{1 . .}$ and $N_{2 i j} / N_{2 .}$, for the female and male populations, respectively.

According to Das Gupta (1991, 1993), the cell proportions, $N_{1 i j} / N_{1 . .}$ and $N_{2 i j} / N_{2 . .}$, in Equations 1 and 2 can be written as

$$
\frac{N_{1 i j}}{N_{1 . .}}=\left(\frac{N_{1 i j} N_{1 i .}}{N_{1 . j} N_{1 . .}}\right)^{\frac{1}{2}}\left(\frac{N_{1 i j} N_{1 . j}}{N_{1 i .} N_{1 . .}}\right)^{\frac{1}{2}}=A_{1 i j} B_{1 i j}
$$

and

$$
\frac{N_{2 i j}}{N_{2 . .}}=\left(\frac{N_{2 i j} N_{2 i .}}{N_{2 . j} N_{2 . .}}\right)^{\frac{1}{2}}\left(\frac{N_{2 i j} N_{2 . j}}{N_{2 i .} N_{2 . .}}\right)^{\frac{1}{2}}=A_{2 i j} B_{2 i j},
$$

where the ratios $A_{1 i j}$ and $B_{1 i j}$ represent the female IDU population distribution, whereas $A_{2 i j}$ and $B_{2 i j}$ represent the male IDU population distribution, by Factor 1 and Factor 2 , respectively. The difference in the HIV seropositivity rates between the male and the female IDU populations can be expressed as

$$
\begin{aligned}
T_{2 . .}-T_{1 . .}= & (\text { Factor } 1 \text { effect }) \\
& +(\text { Factor } 2 \text { effect })+(\text { rate effect }) \\
= & {\left[F_{1}\left(\bar{A}_{2}\right)-F_{1}\left(\bar{A}_{1}\right)\right]+\left[F_{2}\left(\bar{B}_{2}\right)-F_{2}\left(\bar{B}_{1}\right)\right] } \\
& +\left[R\left(\bar{T}_{2}\right)-R\left(\bar{T}_{1}\right)\right],
\end{aligned}
$$

where

$$
\begin{aligned}
& F_{1}\left(\bar{A}_{2}\right)=\sum_{i j} \frac{T_{1 i j}+T_{2 i j}}{2} \frac{B_{1 i j}+B_{2 i j}}{2} A_{2 i j}, \\
& F_{2}\left(\bar{B}_{2}\right)=\sum_{i j} \frac{T_{1 i j}+T_{2 i j}}{2} \frac{A_{1 i j}+A_{2 i j}}{2} B_{2 i j},
\end{aligned}
$$

and

$$
R\left(\bar{T}_{2}\right)=\sum_{i j} \frac{\frac{N_{2 i j}}{N_{2 .}}+\frac{N_{1 i j}}{N_{1 . .}}}{2} T_{2 i j}
$$

In Equation 6, the rates (i.e., age- and ethnicity-specific rates) and Factor 2 (i.e., ethnic composition) are standardized, whereas the rates and Factor 1 (i.e., age structure) are standardized in Equation 7, for the male IDU population. Both age structure and ethnic composition are standardized in Equation 8, which leads to an HIV seropositivity rate for the male IDU population, adjusted for confounding factor effects. The corresponding standardized rates, $F_{1}\left(\bar{A}_{1}\right), F_{2}\left(\bar{B}_{1}\right)$, and $R\left(\bar{T}_{1}\right)$, for the female IDU population can be calculated in the same way.

The difference in the crude HIV seropositivity rates between the male and the female IDU populations can, therefore, be decomposed into three component effects: (1) the Factor 1 effect $\left[F_{1}\left(\bar{A}_{2}\right)-F_{1}\left(\bar{A}_{1}\right)\right]$, which is attributed to the different age structures in the two populations; (2) the Factor 2 effect $\left[F_{2}\left(\bar{B}_{2}\right)-F_{2}\left(\bar{B}_{1}\right)\right]$, which is attributed to the different ethnic compositions; and (3) the rate effect $\left[R\left(\bar{T}_{2}\right)-R\left(\bar{T}_{1}\right)\right]$, which is attributed to the different factor-specific rates.

More than two factors also can be simultaneously handled in standardization and decomposition to take other compositional factors into account. The general formulas for the standardization and decomposition for $P$ factors and multiple populations are given by Das Gupta (1991, 1993).

At the time of this study, none of the existing decomposition methods provides the standard error estimates of the component effects that would enable significance tests when sample data are analyzed. Mathematic derivations of the standard errors of the component effects may be possible on the basis of linearization (delta method), but this is not easy. Thanks to the development of computer hardware and software, the nonparametric bootstrapping method (Efron, 1979, 1981; Efron \& Tibshirani, 1986; Miller, 1974; Mooney \& Duval, 1993) can be applied in such a way as to estimate standard errors of the component effects empirically. Bootstrapping uses a computer to draw resamples from the original sample randomly and repetitively with replacement and with the same size of the original sample. Once the pool of the bootstrap resamples has been established, the sampling distribution of the component effect estimates can be created, and the standard errors and confidence intervals of the component effects can be estimated for significance 
testing. Various bootstrapping methods can be applied for the purpose, and most of them do not require any sampling distribution assumption (Mooney \& Duval, 1993). However, when it is plausible to assume that the sampling distribution of a population parameter estimate is normally distributed but there is no analytic standard error formula available for it, the bootstrapping normal approximation method, which typically requires much fewer bootstrapping resamples than other bootstrapping methods, can be used to estimate the standard error (Mooney \& Duval, 1993).

Let us denote $\hat{\theta}_{b}$ as the component effect estimated from one of the bootstrapping resamples; thus, the distribution of $\hat{\theta}_{b}$ is an estimate of the sampling distribution of the component effect. The standard deviation of the sampling distribution associated with the mean value of $\hat{\theta}_{b}$ is

$$
\hat{\sigma}_{\hat{\theta}}=\left\{\left[\sum\left(\hat{\theta}_{b}-\hat{\theta}_{(.)}\right)^{2}\right] /(B-1)\right\}^{1 / 2},
$$

where $B$ is the total number of bootstrapping resamples, and the mean value of $\hat{\theta}_{b}$ is

$$
\hat{\theta}_{(.)}=\sum \hat{\theta}_{b} / B \text {. }
$$

As $B \Rightarrow \infty$, the standard deviation of the sampling distribution approaches the standard error of the component effect. With the bootstrapping normal approximate method, it has been shown that little improvement in the approximation occurs as $B$ exceeds $50-200$ (Efron \& Tibshirani, 1986). In other words, a limited number of bootstrapping resamples (i.e., 50-200) would be enough for standard error estimation if the sampling distribution of the component effects is approximately normal. Fortunately, because each component effect estimate is, in fact, the estimated difference between two adjusted rates, the sampling distribution of the component effects should be approximately normal in theory. Nevertheless, the normality of the component effects was tested in this study by applying the $\mathrm{Q}-\mathrm{Q}$ normal probability plots before the bootstrapping normal approximation method was employed to estimate the standard errors of the component effects.

\section{COMPUTER PROGRAM}

The authors have developed a Windows-based computer program, DECOMP, to conduct standardization and decomposition, as well as to estimate the standard errors of the component effects by applying the bootstrapping normal approximation method (a free copy of the program can be downloaded from http://www.wright.edu/ jwang/). Although DECOMP currently provides Das

Outcome Measure Difference Standardization and Decomposition

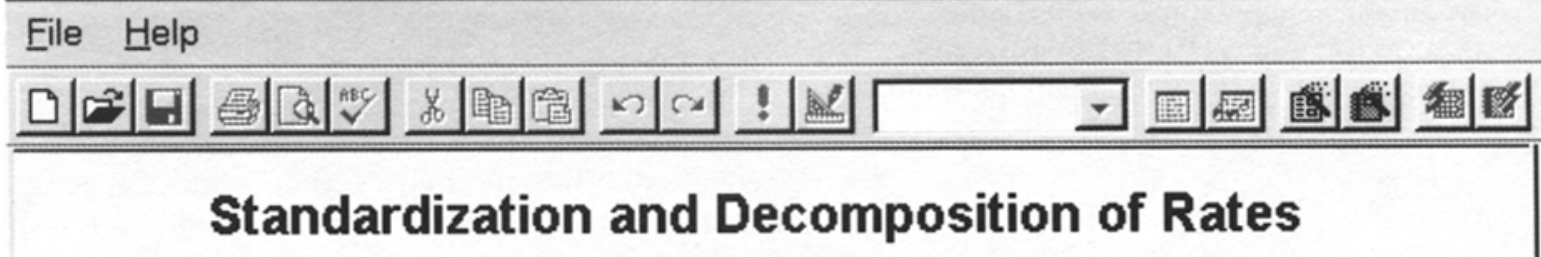

(DECOMP V. 1.0 July 1998)

Individual Data

Grouped Data

Exit

Figure 1. Starting the DECOMP program. 
Gupta's (1991, 1993) and Kitagawa's (1955) methods plus bootstrapping, a future version will include other standardization and decomposition methods.

DECOMP is a user friendly program. Here we briefly explain how to use DECOMP to run standardization and decomposition analysis with the help of Figures 1-4. Figure 1 is the starting window of DECOMP. In this window, the type of data is specified. DECOMP can use either individual data or grouped data (i.e., a contingency table). Individual data must be used if bootstrapping is desired. However, DECOMP allows the user to convert a grouped data file into an individual data file if the outcome measure in the data is a rate, percentage, or proportion.

After the type of data is selected in the starting window, the window of "Program Settings," shown in Figure 2, will appear on screen. The user can select either a DBF or an ASCII file format for data input. If an ASCII file is input, it can be saved as a DBF file for later use. The user can view and alter the data structure at this time. Selecting "Import Selected File" loads the data into the program. The next step is then to specify the number of factors for analysis (two, in our example) and to specify the variables that define population, outcome, and factors appropriately. In our example, the population variable is "Pop," the outcome measure is "Rate," and Factors 1 and
2 are "Agegroup" and "Race," respectively. Note that the values of the population variable "Pop" used to define Populations 1 and 2 are arbitrary. One can click on the "Reverse" icon to switch population definition (see Figure 3).

If an estimation of the standard errors of the component effects is desired, the "Run Bootstrapping" option can be selected. The program allows the user to specify the number of bootstrapping resamples. When the sample size is too large, the program allows the user to draw a smaller sample randomly from the original large sample for analysis.

Figure 4 shows the results of bootstrapping, standardization, and decomposition with 200 resamples.

\section{RESULTS}

The HIV seropositivity rates for the male and female IDU populations in the northeastern United States are shown by ethnicity and age groups in Table 1 . The HIV seropositive rate among IDUs was high (overall, 36.73\%) in this sample. Corresponding rates for the male and female IDUs were $37.39 \%$ and $34.60 \%$, respectively. The difference (about $2.79 \%$ ) is statistically significant $(t=$ $2.10, d f=7,376, p=.0358$ ). Table 1 shows that the ageand ethnicity-specific HIV seropositivity rates differ be-

Program Settings (Individual Data)

Input data file :

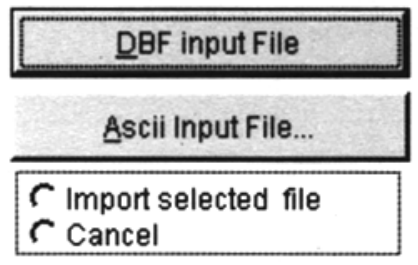

Select number of factors for analysis:
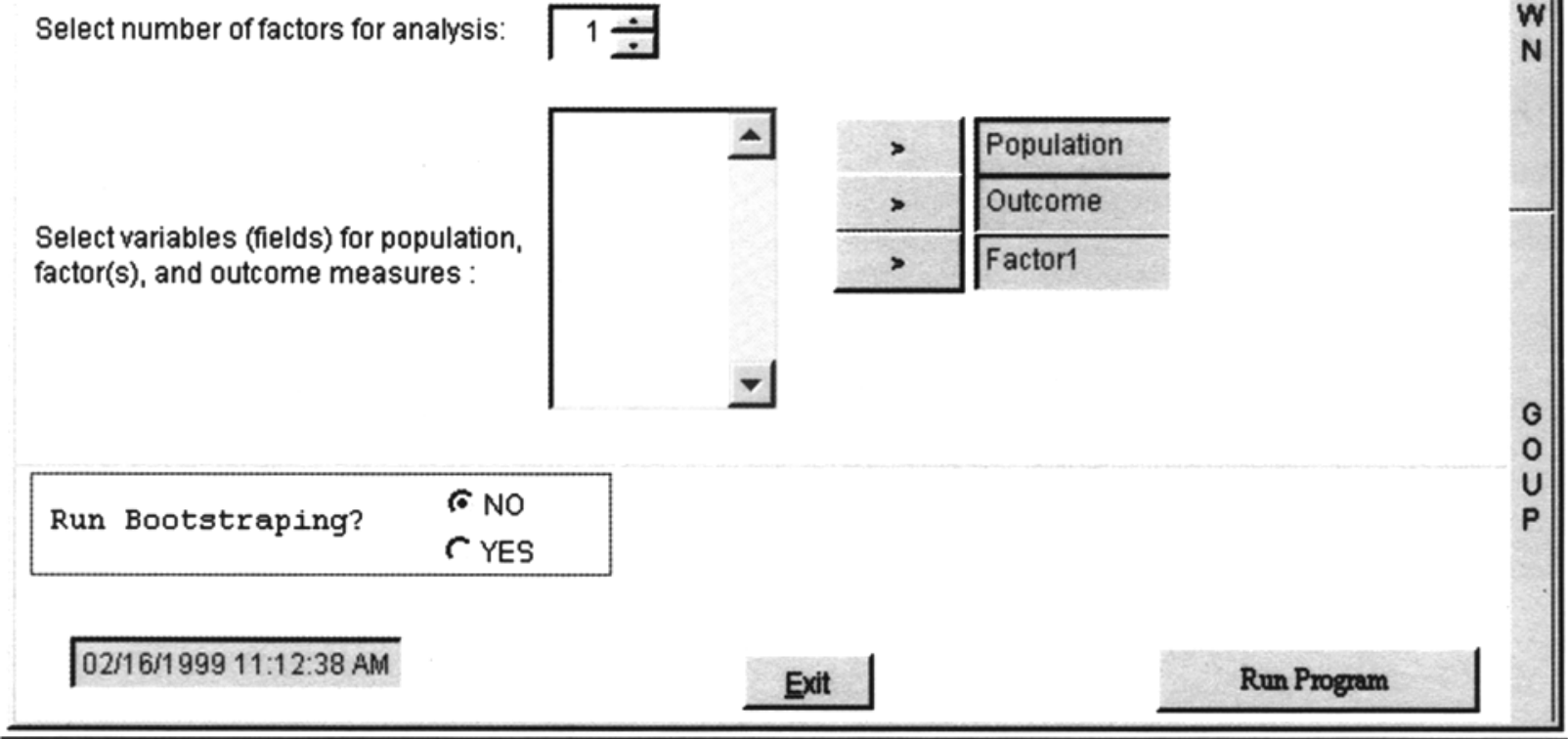


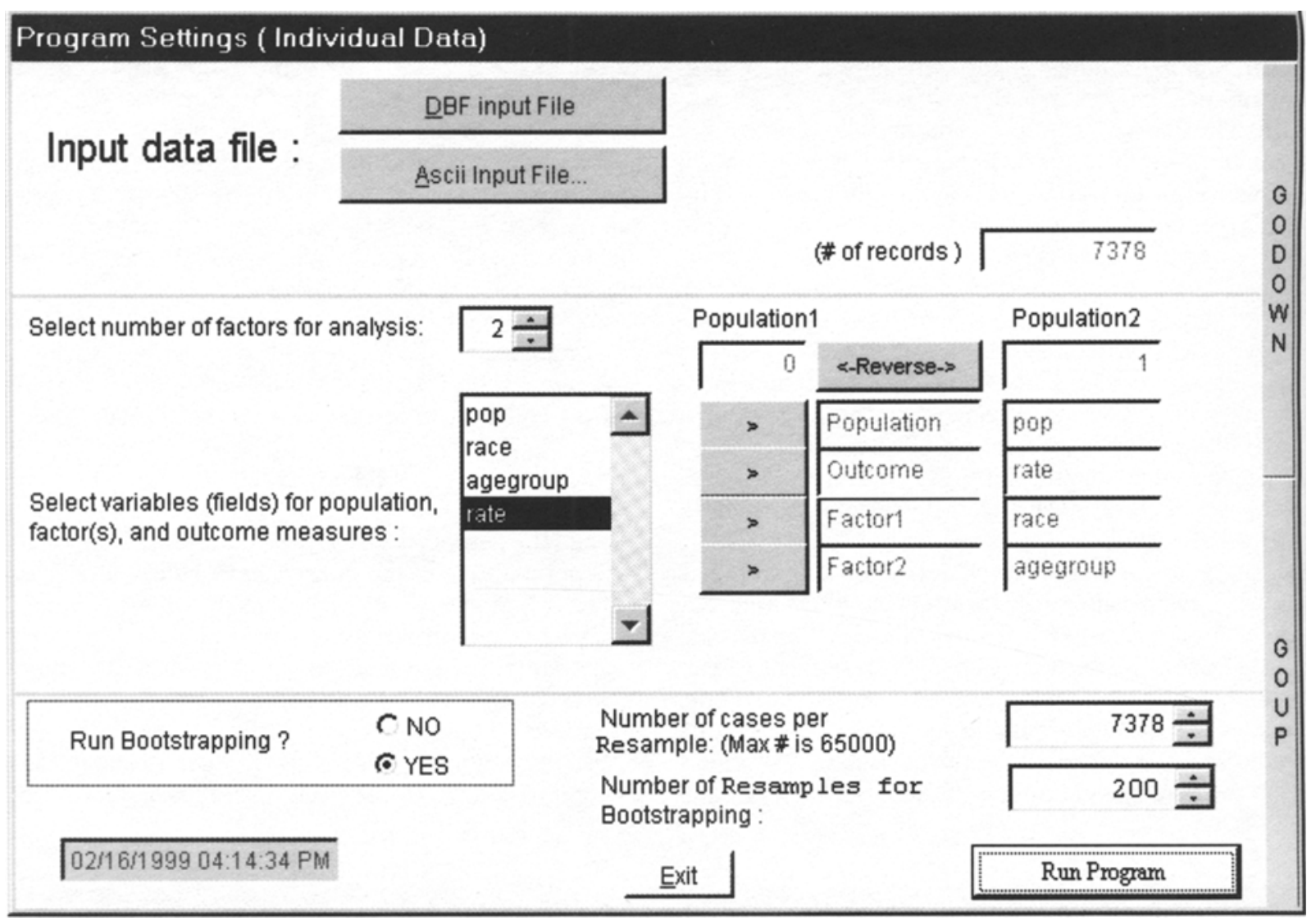

Figure 3. Standardization, decomposition, and bootstrapping.

tween the male and the female IDU populations and that the distributions of IDUs among these two populations also vary by age and ethnic groups. Therefore, to com-

Table 1

Number of Injection Drug Users (IDUs) and HIV Seropositive Rate (\%) by Age and Ethnicity, Northeastern Region of the United States, 1988-1991

\begin{tabular}{|c|c|c|c|c|c|}
\hline \multirow[b]{3}{*}{$\begin{array}{c}\text { Ethnicity* } \\
(j)\end{array}$} & \multirow[b]{3}{*}{$\begin{array}{c}\text { Age Group } \\
(i)\end{array}$} & \multicolumn{4}{|c|}{ Population } \\
\hline & & \multicolumn{2}{|c|}{ Male IDUs } & \multicolumn{2}{|c|}{ Female IDUs } \\
\hline & & $\begin{array}{l}\text { Size } \\
\left(n_{i j}\right)\end{array}$ & $\begin{array}{l}\text { Rate } \\
\left(T_{i j}\right)\end{array}$ & $\begin{array}{l}\text { Size } \\
\left(n_{i j}\right)\end{array}$ & $\begin{array}{l}\text { Rate } \\
\left(T_{i j}\right)\end{array}$ \\
\hline \multirow[t]{4}{*}{1 (black) } & $1(<25)$ & 130 & 12.31 & 70 & 17.14 \\
\hline & $2(25-34)$ & 1,305 & 34.90 & 604 & 35.55 \\
\hline & $3(35-44)$ & 1,539 & 52.91 & 428 & 48.71 \\
\hline & $4(45+)$ & 316 & 44.44 & 43 & 55.81 \\
\hline \multirow[t]{4}{*}{2 (Hispanic) } & $1(<25)$ & 211 & 16.67 & 55 & 14.55 \\
\hline & $2(25-34)$ & 697 & 36.40 & 127 & 39.37 \\
\hline & $3(35-44)$ & 334 & 51.20 & 44 & 32.56 \\
\hline & $4(45+)$ & 48 & 41.67 & 9 & 55.56 \\
\hline \multirow[t]{4}{*}{3 (white) } & $1(<25)$ & 105 & 12.38 & 72 & 22.22 \\
\hline & $2(25-34)$ & 475 & 19.20 & 178 & 20.34 \\
\hline & $3(35-44)$ & 424 & 21.23 & 103 & 13.59 \\
\hline & $4(45+)$ & 49 & 12.50 & 12 & 8.33 \\
\hline Total & & 5,633 & 37.39 & 1,745 & 34.60 \\
\hline
\end{tabular}

Note-Data source: The national database of the National Institute on Drug Abuse's National AIDS Demonstration Research (NADR) projects. *Only "black, Hispanic, and white IDUs were selected for the analysis. pare the HIV seropositivity rates between the two populations, age structure and ethnic composition were standardized across the two populations. Furthermore, the difference in the HIV seropositivity rates between the two IDU populations was decomposed into three components: (1) the Factor 1 effect, attributed to different age structures; (2) the Factor 2 effect, attributed to different ethnic compositions; and (3) the rate effect, attributed to different factor-specific rates in the two populations.

The results of standardization and decomposition are shown in Table 2. Under the "Standardization" column, the figures in the first row ("Factor 1 Effect") are the adjusted HIV seropositivity rates for male and female IDUs, respectively, after Factor 2 (i.e., ethnic composition) and age- and ethnicity-specific rates are standardized. The second row ("Factor 2 Effect") shows the adjusted HIV seropositivity rates after Factor 1 (i.e., age structure) and age- and ethnicity-specific rates are standardized. The third row ("Rate Effect") shows the adjusted rates by standardizing both Factors 1 and 2.

The "Decomposition" column in Table 2 presents the differences in figures between columns 2 and 3 . They are Factor 1 effect $=1.93$, Factor 2 effect $=-0.04$, and rate effect $=0.90$, respectively. The difference in the last row (2.79) under the same column is the difference in the crude HIV seropositivity rates between the male and the female IDUs. Figures in the last column of Table 2 are 


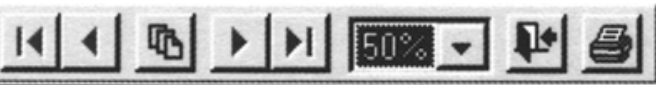

RATE DECOMPOSITION FROGRAK STATISTICS

Two Factor

Date: $11 / 17 / 199$

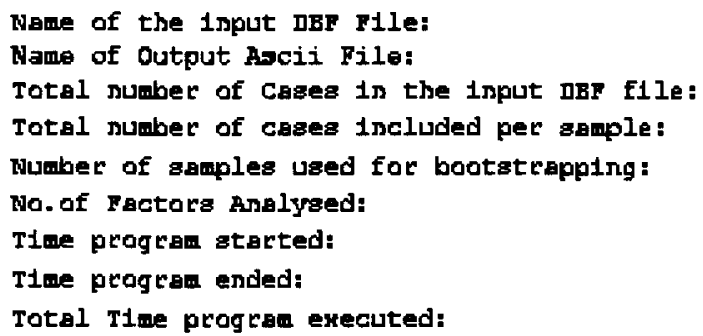

D: \DECONP DATA \DECON9AMIC. DEF

. F.

7378

7378

200

2.0

$09: 33: 03$

09: 36: 39

4 min 35.5 sec

Results of standacdization and Decomposition ( Two Factora)

(STANDARDISATION)

\begin{tabular}{|c|c|c|c|c|}
\hline & Bopulation 2 & Bopulation 1 & $\begin{array}{l}\text { DIfrereoce } \\
\text { iorfectas }\end{array}$ & $\begin{array}{c}\text { dictribution } \\
\text { of effecte }\end{array}$ \\
\hline $\begin{array}{c}\text { Factor I Effact: } \\
\text { (Standard Error) }\end{array}$ & 0.366001 & 0.346619 & $\begin{array}{c}0.019381 \\
(0.002909) \\
\end{array}$ & $69.05 \%$ \\
\hline $\begin{array}{c}\text { Factor } 2 \text { Effect: } \\
\text { (Standard Error) }\end{array}$ & 0.356210 & 0.356410 & $\begin{array}{l}-.000200 \\
(0.003003) \\
\end{array}$ & $-0.71 \%$ \\
\hline $\begin{array}{l}\text { Rate Effect: } \\
\text { (Standard Error) }\end{array}$ & 0.361238 & 0.352354 & $\begin{array}{l}0.008884 \\
(0.012645)\end{array}$ & $31.65 \%$ \\
\hline $\begin{array}{l}\text { Crude Rate: } \\
\text { [standard Error] }\end{array}$ & 0.372784 & 0.344719 & $\begin{array}{l}0.028065 \\
(0.012631)\end{array}$ & $100.00 \%$ \\
\hline
\end{tabular}

Figure 4. Program output.

the percentage distribution of the component effects, which shows the relative contribution of each component effect to the difference in the crude rates. The percentages sum to $100 \%$. A positive percentage indicates the proportion that the corresponding component effect contributes to the rate difference. A negative percentage indicates the extent to which the corresponding component effect tends to narrow the rate difference. In the example, the Factor 1 effect accounts for about $68.97 \%$ of the difference in the crude HIV seropositive rate. The Factor 2 effect tends to narrow the difference in the crude rate (percent-

Table 2

Results of Standardization and Decomposition

\begin{tabular}{|c|c|c|c|c|}
\hline \multirow[b]{3}{*}{$\begin{array}{l}\text { Component } \\
\text { Effect }\end{array}$} & \multirow{2}{*}{\multicolumn{2}{|c|}{ Standardization }} & \multicolumn{2}{|c|}{ Decomposition } \\
\hline & & & \multirow[b]{2}{*}{$\begin{array}{c}\text { Difference } \\
\text { (Effect) }\end{array}$} & \multirow{2}{*}{$\begin{array}{c}\text { Percent } \\
\text { Distribution } \\
\text { of Effect }\end{array}$} \\
\hline & $\begin{array}{c}\text { Male } \\
\text { (Pop2) }\end{array}$ & $\begin{array}{l}\text { Female } \\
\text { (Popl) }\end{array}$ & & \\
\hline Factor 1 effect & 36.73 & 34.81 & 1.93 & 68.97 \\
\hline Factor 2 effect & 35.75 & 35.79 & -0.04 & -1.39 \\
\hline Rate effect & 36.26 & 35.36 & 0.90 & 32.41 \\
\hline Crude rate & 37.39 & 34.60 & 2.79 & 100.00 \\
\hline
\end{tabular}

age distribution of the effect is $-1.39 \%$ ). The rate effect counts for about $32.41 \%$ of the crude rate difference.

The distribution normality of the component effects estimated from bootstrapping with 200 resamples was tested by using Q-Q normal probability plots. In each plot shown in Figures 5-7, the plot points cluster around a straight line, indicating that the bootstrapping estimated values of each component effect are from a normal distribution.

The component effects and their standard errors were estimated from the bootstrapping normal approximation method for various number of resamples changed from 50 to 1,000 , with an increment of 50 resamples each time. The component effect estimates and their standard errors are very stable, particularly when the number of resamples exceeds 150 . This provides a support of Efron and Tibshirani's (1986) finding. We would, therefore, suggest that $150-200$ bootstrapping resamples would be enough to estimate standard errors of the component effects.

In Table 3 we provide the results of standardization and decomposition on the basis of 200 bootstrapping resamples. In addition to the component effect estimates, 


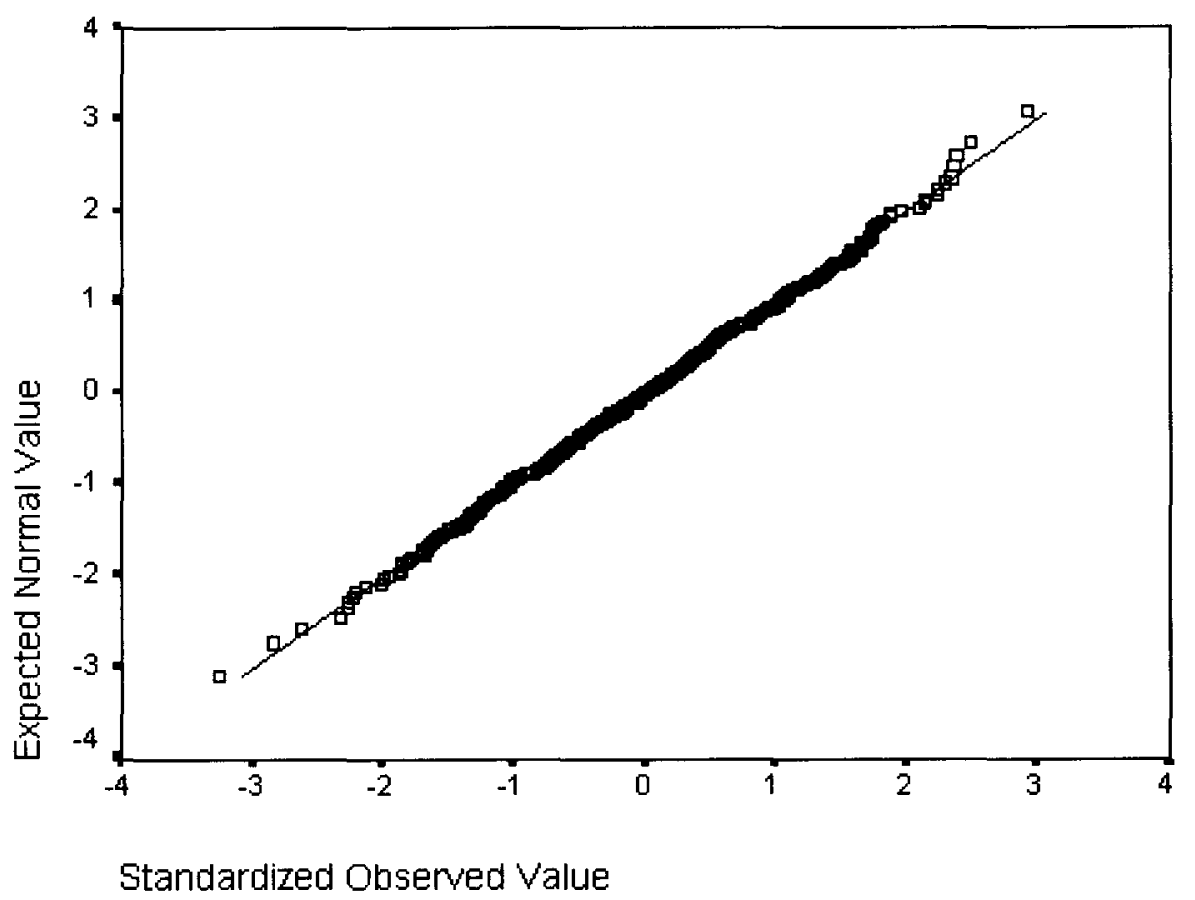

Figure 5. Normal Q-Q plot of adjusted rate.

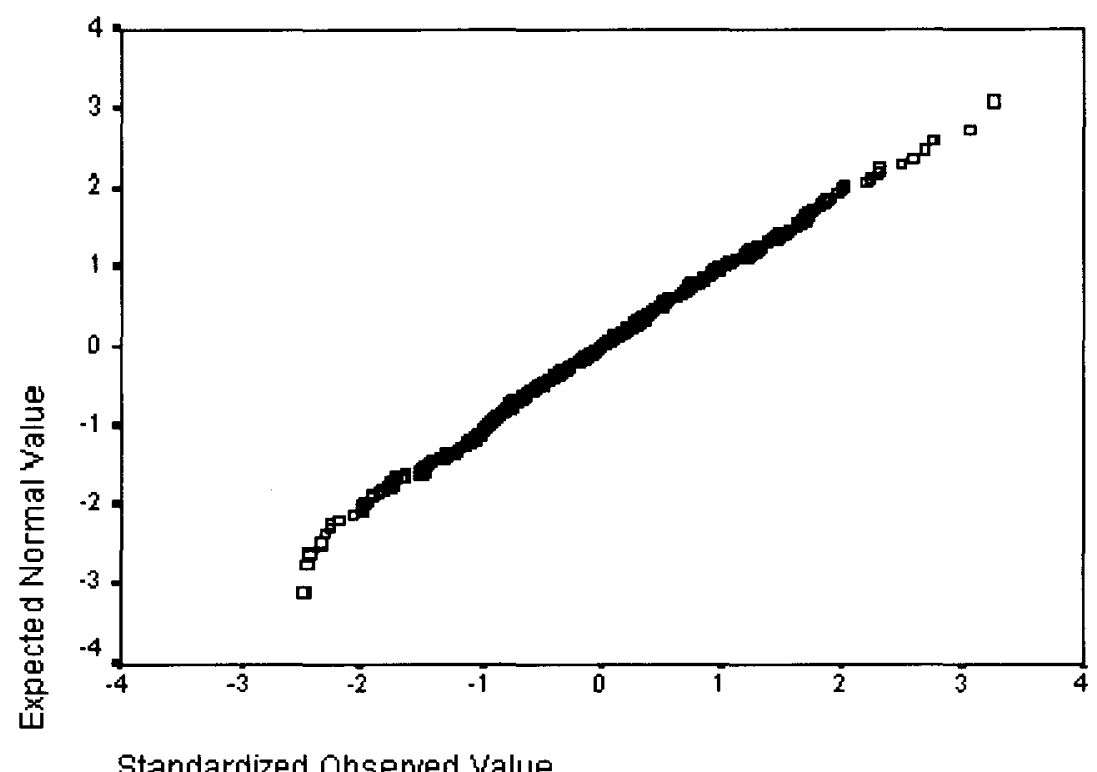

Figure 6. Normal Q-Q plot of Factor 1 effect.

which are very close to those in Table 2, the standard error of each component effect is also reported. With standard errors available, we are able to conduct significance tests for the component effects. Among the three component effects, only the Factor 1 effect (i.e., the effect attributed to different age structures) is statistically significant $(t=6.69, d f=199, p<.001)$. Neither the Factor 2 effect (i.e., the effect attributed to different ethnic com- positions) nor the rate effect (i.e., the effect attributed to the different age- and ethnicity-specific rates) were statistically significant $(t=-0.07, d f=199, p=.9443$, and $t=0.70, d f=199, p=.4847$, respectively). Their contributions to the observed rate difference may be nothing more than chance. The fact that only the Factor 1 effect contributes significantly to the observed rate difference implies that there would be no difference in the HIV 


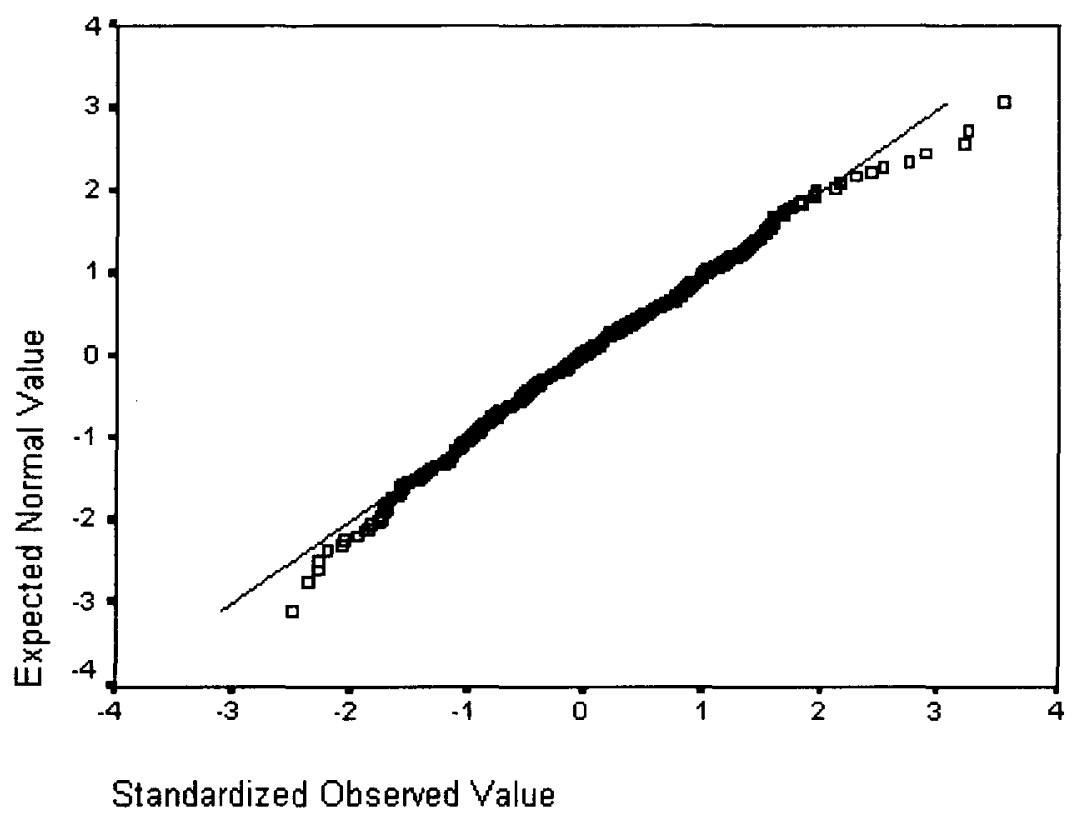

Figure 7. Normal Q-Q plot of Factor 2 effect.

seropositivity rates between the male and the female IDU populations in the northeastern United States if the age structures were the same among the two populations.

\section{DISCUSSION}

Standardization and decomposition are widely used for comparing rates, such as birth rates, death rates, or unemployment rates, between different groups or populations in population studies. In fact, the methods can be applied to a very wide ranges of measures. Any measure that yields an expected number of events or an expected total amount of some variable for the population when multiplied by the number of cases in the population is appropriate for such application. Rates, percentages, proportions, ratios, and arithmetic means satisfy this requirement (Kitagawa, 1964). Behavior and health research could benefit from the application of standardization and decomposition techniques.

Table 3

Results of Bootstrapping, Standardization, and Decomposition

\begin{tabular}{|c|c|c|c|c|c|}
\hline \multirow{4}{*}{$\begin{array}{c}\text { Component } \\
\text { Effect }\end{array}$} & \multirow{2}{*}{\multicolumn{2}{|c|}{ Standardization }} & \multicolumn{3}{|c|}{ Decomposition } \\
\hline & & & \multirow{2}{*}{\multicolumn{2}{|c|}{$\begin{array}{c}\text { Difference } \\
\text { (Effect) }\end{array}$}} & \multirow{3}{*}{ 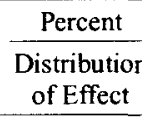 } \\
\hline & \multirow{2}{*}{$\begin{array}{c}\text { Male } \\
\text { (Pop2) }\end{array}$} & \multirow{2}{*}{$\begin{array}{l}\text { Female } \\
\text { (Pop1) }\end{array}$} & & & \\
\hline & & & Dif & $S E$ & \\
\hline Factor 1 effect & 36.60 & 34.66 & 1.94 & 0.29 & 69.05 \\
\hline Factor 2 effect & 35.62 & 35.64 & -0.02 & 0.30 & -0.71 \\
\hline Rate effect & 36.12 & 35.24 & 0.88 & 1.26 & 31.65 \\
\hline Crude rate* & 37.28 & 34.47 & 2.81 & 1.26 & 100.00 \\
\hline
\end{tabular}

Note-Standard errors were estimated by bootstrapping on the basis of 200 resamples. *Estimated population crude rates.
However, we should keep in mind that "the problem of decomposition of the difference between two crude rates into several additive effects is different from the problem of, and cannot be adequately handled by, regression analysis" (Das Gupta, 1993, p. 2). This is because the difference between two crude rates is not the equivalent of a concept like total variance of a dependent variable in regression analysis (Kitagawa, 1955). A variable may play an important role in explaining the variation of a dependent variable in regression but may turn out not to be important in explaining the difference between two crude rates. For example, ethnicity is often found to be a significant factor for explaining the variation of a behavioral measure in regression analysis. But ethnicity may contribute nothing to explaining the difference in the prevalence rate of the behavior between two populations if the two populations have approximately the same ethnic composition.

When decomposition analysis is conducted by using sample rather than population data, it is desirable to take the uncertainty into account. In such a case, the standard errors of the component effects need to be estimated for significance tests. According to the best of our knowledge, this study is the first to estimate standard errors of the component effects and to conduct significance tests for them. Our analytic results provide evidence that the sampling distribution of the component effects is approximately normal; therefore, the bootstrapping normal approximation method can be used to estimate the standard errors of the component effects with a limited number (i.e., 150-200) of resamples. As a result, it can substantially reduce the computing time for bootstrapping. 


\section{REFERENCES}

Bickel, P. J., Hammel, E. A., \& O'Connell, J. W. (1975). Sex bias in graduate admissions: Data from Berkeley. Science, 187, 398-404.

BongaARTS, J. (1978). A framework for analyzing the proximate determinants of fertility. Population \& Development Review, 4, 105-132.

BRown, B. S., \& BESCHNER, G. M. (1993). Handbook on risk of AIDS: Injection drug users and sexual partners. Westport, CT: Greenwood.

Cho, L. J., \& RETHERford, R. D. (1973). Comparative analysis of recent fertility trends in East Asia. Proceedings of IUSSP International Population Conference, 2, 163-181.

ClogG, C. C. (1978). Adjustment of rates using multiplicative models. Demography, 15, 523-539.

Clogg, C. C., \& Eliason, S. R. (1988). A flexible procedure for adjusting rates and proportions, including statistical method for group comparisons. American Sociological Review, 53, 267-283.

Clogg, C. C., \& Shockey, J. W. (1985). The effect of changing demographic composition on recent trends in underemployment. Demography, 22, 395-414.

Clogg, C. C., Shockey, J. W., \& Eliason, S. R. (1990). A general statistical framework for adjustment of rates. Sociological Methods \& Research, 19, 156-195.

Das GuPTA, P. (1991). Decomposition of the difference between two rates and its consistency when more than two populations are involved. Mathematical Population Studies, 3, 105-125.

DAs GuPTA, P. (1993). Standardization and decomposition of rates: $A$ users's manual (U.S. Bureau of the Census, Current Population Reports, Series P23-186). Washington, DC: U.S. Government Printing Office.

EFron, B. (1979). Bootstrap methods: Another look at the Jackknife. Annals of Statistics, 7, 1-26.

EFroN, B. (1981). Nonparametric standard errors and confidence intervals [with discussion]. Canadian Journal of Statistics, 9, 139-172.
EFron, B., \& TibshiRANi, R. (1986). Bootstrap methods for standard errors, confidence intervals, and other measures of statistical accuracy. Statistical Science, 1, 54-77.

Kim, Y. J., \& Strobino, D. M. (1984). Decomposition of the difference between two rates with hierarchical factors. Demography, 15,99-112.

KiTAGaWA, E. M. (1955). Components of a difference between two rates. Journal of the American Statistical Association, 50, $1168-1194$.

KITAGaWA, E. M. (1964). Standardized comparisons in population research. Demography, 1, 296-315.

LIAO, T. F. (1989). A flexible approach for the decomposition of rate differences. Demography, 26, 717-726.

Miller, R. G. (1974). The Jackknife: A review. Biometrika, 61, 1-15.

Mooney, C. Z., \& Duval, B. D. (1993). Bootstrapping: A nonparametric approach to statistical inference. Newbury Park, CA: Sage.

Nathanson, C. A., \& KIM, Y. J. (1989). Components of change in adolescent fertility. Demography, 26, 85-98.

Pullum, T. W. (1978). Standardization (World Fertility Survey Technical Bulletins, No. 597). Voorburg, The Netherlands: International Statistical Institute.

Pullum, T. W., Tedrow, L. M., \& Herting, J. R. (1989). Measuring change and continuity in parity distributions. Demography, 26, 485-498.

UNITED NATIONS (1979). The methodology of measuring the impact of family planning programs (Manual IX, Population Studies, No. 66). New York: Author.

Wojtkiewicz, R. A., Mclanahan, S. S., \& Garfinkel, I. (1990). The growth of families headed by women: 1950-1980. Demography, 27, 19-30.

(Manuscript received April 5, 1999; revision accepted for publication November 13, 1999.) 